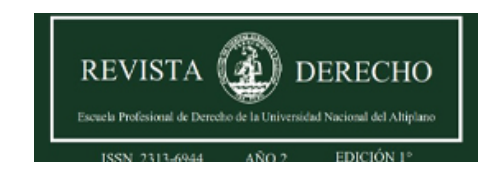

Revista de Derecho

ISSN: 2313-6944

ISSN: 2707-9651

revistaderecho@unap.edu.pe

Universidad Nacional del Altiplano

Perú

\title{
Violencia familiar y trastorno depresivo en mujeres e integrantes del grupo familiar en la jurisdicción de la Comisaría de Familia de Lima: Una revisión sistemática de artículos científicos disponibles en acceso abierto en español indexados en Scielo
}

Rivera Fernández, Dorothy Herfilia; Benavides Román, Alexander Masías; Chávez Yomona, Marleny Violencia familiar y trastorno depresivo en mujeres e integrantes del grupo familiar en la jurisdicción de la Comisaría de Familia de Lima: Una revisión sistemática de artículos científicos disponibles en acceso abierto en español indexados en Scielo

Revista de Derecho, vol. 7, núm. 1, 2022

Universidad Nacional del Altiplano, Perú

Disponible en: https://www.redalyc.org/articulo.oa?id=671870939005

DOI: https://doi.org/10.47712/rd.2022.v7i1.163

\section{(c) (1)}

Esta obra está bajo una Licencia Creative Commons Atribución 4.0 Internacional 


\section{Violencia familiar y trastorno depresivo en mujeres e integrantes del grupo familiar en la jurisdicción de la Comisaría de Familia de Lima: Una revisión sistemática de artículos científicos disponibles en acceso abierto en español indexados en Scielo}

Family violence and depressive disorder in women and members of the family group in the jurisdiction of the Family Police Station of Lima: A systematic review of scientific articles available in open access in Spanish indexed in Scielo

Dorothy Herfilia Rivera Fernández

Universidad César Vallejo, Perú

driverafe@ucv.edu.pe

(iD https://orcid.org/0000-0003-0530-5084

Alexander Masías Benavides Román

Universidad César Vallejo, Perú

benavidesro22@ucvvirtual.edu.pe

(D) https://orcid.org/0000-0003-0398-938X

Marleny Chávez Yomona

Universidad César Vallejo, Perú

marleyesi@hotmail.com

(D) https://orcid.org/0000-0001-6507-1422
DOI: https://doi.org/10.47712/rd.2022.v7i1.163

Redalyc: https://www.redalyc.org/articulo.oa?

$\mathrm{id}=671870939005$

Recepción: 11 Noviembre 2021

Aprobación: 27 Diciembre 2021

Publicación: 04 Enero 2022

\section{Resumen:}

El objetivo del presente artículo es sistematizar evidencia científica disponible sobre violencia familiar y su relación con los trastornos depresivos. La investigación estuvo orientada a la indagación bibliográfica en la base de datos de Scielo. Se enfocó en la temática de violencia intrafamiliar y generación de la afección mental, especialmente los trastornos depresivos y pautas para prevenirlos, de utilidad para el quehacer investigativo y asistencial de autoridades, operadores judiciales y profesionales de la salud. Entre los principales resultados se obtuvo que los trastornos depresivos están fuertemente asociados a la violencia intrafamiliar. Asimismo, se estableció que las mujeres, en especial las embarazadas, expuestas a violencia por sus parejas íntimas, sufrieron de maltratos psicológicos (presentando signos depresivos), seguidos de maltratos físicos, violencia sexual y violencia económicapatrimonial; al respecto el MINP (noviembre 2020), señaló que, de 58595 mujeres sometidas a violencia familiar, entre los 18 y 59 años de edad, el $49.8 \%$ era por violencia psicológica. De otro lado, los niños y adolescentes también se vieron afectados en su salud mental a consecuencia de las agresiones ejercidas por sus padres, alterándose sus procesos psicosociales y convirtiéndose en un factor de riesgo para síntomas depresivos u otras conductas autodestructivas. En ese sentido, el MINSA informó que los trastornos más habituales en infantes y jóvenes son por problemas emocionales, como depresión o ansiedad ligados a violencia doméstica. Por último, los adultos mayores integrantes del grupo familiar, también fueron afectados por algún tipo de violencia, sobre todo la psicológica, en especial los adultos entre los 70 y 79 años de edad, que presentaron signos de depresión.

Palabras ClaVE: Violencia familiar, violencia intrafamiliar, violencia doméstica, violencia psicológica, trastorno depresivo.

\section{Abstract:}

This article aims to systematize available scientific evidence on family violence and its relationship with depressive disorders. The research was oriented to the bibliographic investigation in the database of Scielo. It focused on the issue of domestic violence

\section{NotAS DE AUTOR}


and the generation of mental illness, especially depressive disorders and guidelines to prevent them, which are useful for the investigative and assistance work of authorities, judicial operators and health professionals. Among the main results, it was found that depressive disorders are strongly associated with domestic violence. It was also established that women, especially pregnant women, exposed to violence by intimate partners, suffered from psychological abuse (showing depressive signs), followed by physical abuse, sexual violence and economic violence. In this regard, the MINP (November 2020) noted that, of 58595 women subjected to family violence, between 18 and 59 years of age, $49.8 \%$ was due to psychological violence. On the other hand, children and adolescents were also affected in their mental health, as a result of the aggressions carried out by their parents, altering their psychosocial processes and becoming a risk factor for depressive symptoms or other self-destructive behaviors. In this sense, the MINSA reported that the most common disorders in infants and young people are due to emotional problems, such as depression or anxiety linked to domestic violence. Finally, older adults in the family group were also affected by some form of violence, especially psychological violence, especially adults between 70 and 79 years of age, who showed signs of depression.

KEYWORDS: Family violence, domestic violence, domestic violence, psychological violence, depressive disorder.

\section{INTRODUCCIÓN}

El presente artículo está referido a la violencia familiar, que según los psicoterapeutas Bonino y Corsi (2003), es entendida como una relación abusiva, permanente o cíclica que caracteriza el vínculo intrafamiliar donde cualquiera de los miembros del grupo familiar puede convertirse en víctimas (fundamentalmente los miembros más vulnerables del grupo familiar: mujeres, niños y ancianos) o victimarios (por su posición de poder o control: parejas o exparejas sentimentales, padres, madres, o quien haga su función, e hijos) y que viene asociado a la generación de perturbaciones mentales, en especial las asociadas a trastornos depresivos. De modo similar, la Ley 30364 (2015), prescribe que la violencia familiar: "es cualquier acción o conducta que causa muerte, daño o sufrimiento físico, sexual o psicológico y que se produce en el contexto de una relación de responsabilidad, confianza o poder, de parte de un integrante a otro del grupo familiar". Sin duda, la violencia intrafamiliar en la población mundial es un problema que viene en aumento (Cleto et al., 2019); convirtiéndose en un tema de interés y preocupación por entidades nacionales e internacionales (Baena et al., 2020); se estima que un 30\% de mujeres fueron agredidas física o psicológicamente por sus parejas sentimentales (Lara et al., 2019), siendo que los factores socioeconómicos y culturales estaban relacionados al desarrollo de la violencia familiar que los agresores infligen a sus víctimas (Ranielle y Franciéle, 2020), ubicándose como factor determinante de la violencia intrafamiliar, la construcción cultural de identidades totalmente deterioradas (Cudris et al., 2019), tornándose en una cuestión de salud pública y entre sus efectos negativos, repercute en la salud mental de quienes lo padecen, prevaleciendo el trastorno depresivo, que puede llevar a las víctimas de este flagelo a sentir menosprecio por su vida e inclusive a desarrollar ideas suicidas (Brock et al., 2017).

Al respecto, Saldaña y Gorjón (2020) señalaron que la herencia transgeneracional que se trasmite de padres a hijos formaría parte de la estructura de la violencia familiar, la misma que se vuelve cíclica en un entorno familiar que conlleva a la afectación de las interacciones de los individuos. En cuanto a la depresión, es definida por la OMS (2020), como "un desorden mental caracterizado por existencia de tristeza, desinterés o pérdida de placer, sensación de culpa o inadecuada autoestima, patologías del sueño o del apetito, cansancio y con menoscabo en la de concentración”, calculándose que aqueja a más de 300 millones de individuos en el orbe. Sausa (2018) indicó que, en el Perú, según reporte del MINSA del 2016, se aproximó a 2 millones las ocurrencias de depresión y ansiedad que estaban vinculados con conflictos familiares, siendo los menores de 18 años el $70 \%$ del total de pacientes atendidos. García et al. (2016) sostuvo que la violencia también se ejerce en el adulto mayor, y que es generalmente de tipo psicológico, existiendo en la mayoría de hogares, ocasionándoles a estas víctimas depresión y hasta a veces pensamientos de autoeliminación. Con respecto a la ciudad de Lima, la División PNP de Protección contra la Violencia Familiar (2019), informó que se registraron 561 denuncias en la Comisaría de la Familia-Lima, de las cuales el 72\% fueron por agresión psicológica, $7.48 \%$ por agresión 
física, $16.58 \%$ fueron denuncias por violencia física y psicológica, el 1.60\% se debió a agresión sexual y 1.96\% por violencia económica y patrimonial.

La actual investigación es relevante ya que brindará un aporte teórico a la comunidad científica sobre la correspondencia entre violencia familiar y el trastorno depresivo que sufren las víctimas afectadas por este tipo de violencia, conocimientos que podrán ser tomadas en cuenta para elaborar estudios similares usando los aportes citados en este trabajo; así como, dotar de insumos a las autoridades y organismos competentes para desarrollar acciones preventivas y adecuados programas de salud pública ante el peligro de sufrir abusos o eventos violentos en el entorno familiar.

Respecto del objetivo del presente trabajo, se encuentra referido a identificar y examinar sistemáticamente la mejor evidencia científica disponible sobre violencia familiar y los trastornos depresivos presentados en las mujeres o en cualquiera de los miembros del grupo familiar, disponible en la base de datos Scielo, en español y portugués de los últimos 5 años.

\section{METODOLOGÍA}

La investigación contiene una revisión sistemática bajo el enfoque cualitativo, se incluyeron estudios que comprendían a niños y niñas, adolescentes, mujeres y adultos mayores, dentro de la comunidad general, en el contexto de Lima metropolitana, siendo excluidas aquellas revisiones de literatura de naturaleza teórica.

Las categorías de estudio consideradas fueron la violencia intrafamiliar con afección mental y trastornos depresivos en el contexto de una problemática de connotación mundial desarrollados indistintamente en los diferentes grupos socio-culturales, el objetivo de esta breve reseña es presentar un mapa de los conocimientos existentes sobre estos temas, identificando los diseños de estudio aplicados por otros investigadores.

$\mathrm{Al}$ respecto se seleccionaron como resultados primarios los siguientes: estudios de casos con rigor científico, doctrina jurídica, investigaciones trascendentales, así como las experiencias de autores que contribuyeron al éxito del presente artículo de revisión; efectuándose una búsqueda manual empleando las referencias de los estudios primarios y secundarios encontrados en la búsqueda electrónica. Las fuentes de consultas estuvieron relacionadas a artículos de revistas indexadas de los últimos 5 años ubicados en la base de datos de Scielo. Como tesauros se tomó en cuenta la violencia familiar, violencia intrafamiliar, violencia doméstica, violencia psicológica, trastorno depresivo.

El proceso de selección de los estudios fue realizado en 2 fases. La primera se basó en revisar los títulos y resúmenes de las referencias encontradas con nuestra estrategia de búsqueda; se seleccionaron los estudios potencialmente elegibles. El segundo paso consistió en revisar el texto completo de los estudios preseleccionados para confirmar su elegibilidad elaborando una matriz de fichaje en Excel a efectos recolectar y almacenar la información pertinente bajo el método prisma.

\section{RESULTADOS}

Utilizando la base de datos de Scielo se ubicaron 1,040 referencias para la categoría de violencia familiar y 32 referencias para la categoría de trastorno depresivo, que estuviera relacionados con la violencia doméstica o intrafamiliar; considerando referencias con 5 años de antigüedad; de las cuales se descartaron 1,031 referencias de violencia familiar y 26 referencias sobre trastornos depresivos, que luego de revisarse el título y su resumen, no se adecuaron a los criterios de inclusión (primer paso); ahora bien, de las 15 referencias seleccionadas se procedió a su lectura para confirmar la elegibilidad (segundo paso). De la revisión de las 15 referencias de texto completo se excluyeron 7 artículos que no se ajustaban con nuestros criterios de inclusión.

A continuación, se presenta los resultados de los 8 estudios seleccionados de manera descriptiva, anexándose la tabla 1 donde se consignan los resultados de manera sucinta. 
TABLA 1

Autores de textos y revistas indexadas cuyos contenidos fortalecen el tema principal

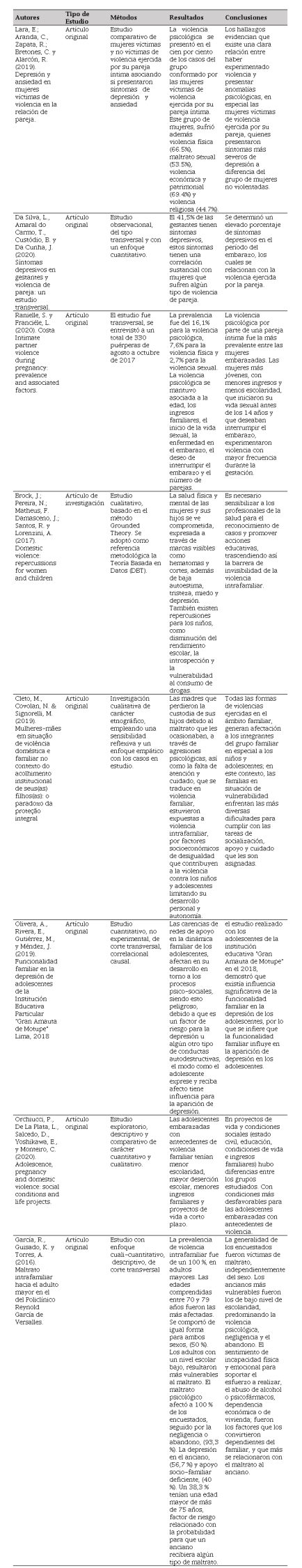


Elaboración propia.

El primer artículo, elaborado por Lara, Aranda, Zapata, Bretones y Alarcón (2019), es un artículo original, desarrollado en República Dominicana, donde la muestra estuvo integrada por 340 mujeres, todas mayores de 18 años, distribuidas en 2 grupos, el primero de mujeres que declararon ser agraviadas por su compañero intimo (VPI) y otro grupo, que manifestaron no haber sido violentadas. El método empleado fue un análisis comparativo de mujeres afectadas por la agresión ejercida por su pareja íntima VPI y las no agredidas; a fin de determinar si las agredidas tienen preeminencia de mostrar síntomas depresivos y de ansiedad. Los resultados fueron que la agresión de tipo psicológica estuvo presente en el 100\% de las ocurrencias del grupo conformado por las víctimas de la agresión ejercida por su compañero íntimo. Este grupo de mujeres, sufrió además violencia física (66.5\%), maltrato sexual (53.5\%), violencia económica y patrimonial $(69.4 \%)$ y violencia religiosa (44.7\%). Como conclusión se tiene que los hallazgos determinan una relación entre violencia y exhibir anomalías psicológicas, en especial las mujeres víctimas de agresión ejercida por su compañero íntimo, quienes presentaron síntomas más severos de depresión a diferencia del grupo de mujeres que manifestaron no haber sido violentadas por sus parejas reportando síntomas de depresión muy leve (Lara et al., 2019). La investigación es relevante en cuanto es la primera de este tipo realizada en poblaciones dominicanas, así como ampliar los conocimientos sobre el bienestar mental de las mujeres inmersas en la violencia intrafamiliar.

La segunda investigación original, expuesta por Da Silva, Amaral do Carmo, Custódio y Da Cunha (2020), realizada en Brasil. Método: es un estudio piloto transversal con la participación de 65 embarazadas. Como resultado se obtuvo que el $41,5 \%$ de las embarazadas tienen síntomas depresivos, que se correlacionan con mujeres que sufren algún tipo de agresión de pareja. Además, los factores como ser soltera, tener educación deficiente, recursos económicos mínimos y gestación no deseada contribuyeron significativamente en la presencia de síntomas depresivos durante la etapa del embarazo. Conclusión: se determinó que existe elevado porcentaje de manifestaciones depresivas en esta fase, los cuales se vinculan con la agresión ejercida por la pareja (Da Silva et al., 2020). El presente estudio es importante en el contexto de la salud pública, donde los profesionales de la salud deberán estar atentos y capacitarse mejor para brindar una adecuada atención a las mujeres gestantes que puedan sufrir de violencia familiar.

El tercer artículo, cuya autoría es de Ranielle y Franciéle (2020), corresponde a un artículo original, efectuado en Cariacica, Brasil. Método: el estudio fue transversal y se realizó en una maternidad donde se entrevistó a un total de 330 puérperas de agosto a octubre de 2017. Para identificar los tipos de violencia se utilizó el instrumento adecuado de la OMS. Resultados obtenidos: La prevalencia fue del 16,1\% para la violencia psicológica, 7,6\% para la agresión física y 2,7\% para la agresión sexual. La violencia psicológica se mantuvo asociada a la edad, los ingresos familiares, el comienzo a la sexualidad, enfermedades presentes en la etapa del embarazo, el deseo de interrumpir el embarazo y el número de parejas. La violencia física se asoció con la escolarización, la iniciación sexual y la enfermedad durante el embarazo. La violencia sexual permaneció asociada con el estado civil y el deseo de interrumpir el embarazo. Conclusiones: La violencia psicológica ejercida por la pareja íntima prevaleció en el grupo de mujeres embarazadas, presentándose con mayor frecuencia esta violencia en mujeres jóvenes, de bajos recursos económicos, poca educación, vida sexual a temprana edad y el deseo de interrumpir el embarazo (Ranielle y Franciéle, 2020). Importancia del estudio: permite evidenciar la ocurrencia de violencia durante el embarazo y la afectación a las mujeres en un momento de fragilidad física y emocional, causando daños a su salud como la depresión que afecta al mismo tiempo al niño recién nacido o por nacer.

El cuarto trabajo de investigación, realizado por Brock, Pereira, Matheus, Damasceno, Santos y Lorenzini (2017), en el estado de Bahía perteneciente a Brasil; tuvo como método de investigación el estudio cualitativo. Se entrevistó a 37 concurrentes de dos juzgados de familia, que componían los grupos de muestra. Resultados: el estudio demostró que, en las mujeres entrevistadas y sus hijos, se vio comprometida la salud, tanto física como mental, expresada a través de signos como hematomas y cortes, disminuida autoestima, tristeza, miedo y depresión. También existen consecuencias para la vida social de las mujeres como resultado del ascetismo 
social y las no condiciones para el mercado laboral, y respecto de los infantes, relacionadas con la merma del rédito escolar, la introspección y la vulnerabilidad al consumo de estupefacientes. Se concluyó en que dada la forma en que la violencia afecta el bienestar y crecimiento humano de los niños, la enfermedad mental de las mujeres se intensifica cuando se sienten culpables por exponer a sus hijos a la violencia doméstica, haciéndose necesario sensibilizar al personal de salud para identificar estos casos y encontrar la mejor solución educativa, trascendiendo el muro invisible de la violencia intrafamiliar (Brock et al., 2017). La importancia de este artículo fue que permitió conocer como las mujeres conceptualizan que efectos genera la violencia doméstica que sufren.

El quinto artículo científico, presentado por Cleto, Covolan y Signorelli (2019), corresponde a una investigación original, realizada en Brasil, con un campo etnográfico que estuvo conformado por instituciones responsables de Servicios de Asistencia Social de Media y Alta Complejidad, El método que utilizaron fue de investigación cualitativa de carácter etnográfico, empleando una sensibilidad reflexiva y un enfoque empático con los casos en estudio; los resultados estuvieron referidos al estudio de casos de madres que perdieron la custodia de sus hijos debido al maltrato que les ocasionaban, a través de agresiones psicológicas, así como la falta de atención y cuidado, que se traduce en violencia familiar. Se tiene que estás madres también estuvieron expuestas a violencia intrafamiliar, teniendo como factor de la violencia los niveles socioeconómicos de desigualdad que contribuyen a la agresión contra infantes y jóvenes limitando su desarrollo personal y autonomía. La conclusión a la que arribaron los autores, entre otras, es que todas las formas de violencias ejercidas en el ámbito familiar, generan afectación a los componentes de la familia, en especial a los niños y adolescentes; en este contexto, las familias en situación de vulnerabilidad enfrentan las más diversas dificultades para cumplir con las tareas de socialización, apoyo y cuidado que les son asignadas (Cleto et al., 2019). La importancia del trabajo se basa en que se evidenció la situación de las madres agraviadas por la violencia intrafamiliar y que convierten, a su vez, en víctimas a sus hijos, los cuales requieren de medidas de protección; así como, los principales desafíos de la red de apoyo del sector estatal.

La sexta investigación, desarrollada por Olivera, Rivera, Gutiérrez y Méndez (2019), consignada como artículo original, efectuado en Perú, utilizó el estudio cuantitativo, no experimental, de corte transversal, correlacional causal. La muestra estuvo compuesta por 91 jóvenes entre los 12 a 17 años de edad, de una institución educativa de Motupe. Según los resultados, se observa carencias de apoyo en la dinámica familiar de los jóvenes, mermando sus procesos psicosociales, favoreciendo a estados depresivos u otras conductas autodestructivas. La parentela es la protección que tiene el ser humano y esta puede transformarse en factor de riesgo cuando aparecen problemas al interior de ella. Como conclusión, se puede aseverar que los jóvenes con menor funcionalidad familiar exhiben mayores signos de depresión (Olivera et al., 2019). Por ende, la importancia de este trabajo de investigación es que nos ayuda a entender que las relaciones conflictivas entre los integrantes del grupo familiar pueden conllevar al adolescente a un estado de depresión que le haga perder el apego a la vida, desarrollando de esta forma ideas suicidas que le permitan finalizar con su dolor emocional.

El sétimo artículo original, producido por Orchiucci, De La Plata, Salcedo, Yoshikawa y Monteiro (2020), realizado en Brasil, se basó en un estudio exploratorio, descriptivo y comparativo de carácter cuantitativo y cualitativo. La muestra estuvo conformada por 3 grupos: el primero de 30 jóvenes no embarazadas, sin antecedentes de agresión; el segundo de 30 jóvenes embarazadas, sin antecedentes de agresión; y el tercero de 30 jóvenes embarazadas, con antecedentes de agresión. Resultados: Las jóvenes del tercer grupo tuvieron bajo índice de instrucción, mayor ausentismo de las escuelas con una economía paupérrima y proyección de metas en breve plazo, en el que no consideraban al matrimonio. Como conclusiones, se observó que, respecto de la condición civil, instrucción, estilo de vida y rentas familiares mostraron una diferencia significativa entre los tres grupos estudiados, denotándose deficientes políticas educativas y sociales para adolescentes embarazadas y víctimas de violencia intrafamiliar; al mismo tiempo, las adolescentes del tercer grupo, indicaron que no tenían proyectos, debido a la violencia doméstica que les limitaba una planificación de vida (Orchiucci et al., 
2020). La relevancia de este trabajo es valorar la relación madre-niño, y que todo tipo de violencia merezca ser estudiado a profundidad a efectos de reforzar las políticas públicas que ayuden a combatir este mal.

Finalmente, el octavo artículo, efectuado por García, R., Guisado, K., y Torres, A. (2016), realizado en la ciudad de Matanzas, Cuba, aplicaron un estudio con enfoque cuali-cuantitativo, descriptivo, de corte transversal. La muestra se conformó por sesenta adultos mayores de 60 años. Los resultados resaltantes fueron: La agresión doméstica en adultos mayores de ambos géneros, con incidencia preminente en un 100 $\%$, siendo los más perjudicados los ancianos entre 70 y 79 años de edad, y se volvían más vulnerables al maltrato, cuanto más bajo era su nivel escolar. El maltrato psicológico afectó al $100 \%$ de los participantes, por negligencia o abandono $93,3 \%$, estado depresivo en el anciano el $56,7 \%$ y deficiente soporte familiar el $40 \%$. Como conclusiones, en general, los participantes fueron víctimas de maltrato, indistintamente del sexo. Los ancianos más desvalidos tenían pocos estudios, prevaleciendo la violencia psicológica y la negligencia. La sensación de discapacidad física y emocional para afrontar las actividades a realizar, el alcoholismo o estupefacientes, subordinación patrimonial o de habitación; fueron los elementos que los transformaron en dependientes del familiar, y que más se vincula con el agravio al anciano (García et al., 2016). La relevancia de la presente investigación es que identifica el maltrato en la familia hacia los ancianos tema que requiere una mayor connotación por el Estado y personal de salud visibilizándolos como integrantes del grupo familiar.

\section{DISCUSIÓN}

El presente estudio tuvo como objetivo sistematizar evidencia científica disponible sobre violencia familiar y su relación con los trastornos depresivos. En efecto, los resultados de los artículos revisados demostraron que las mujeres expuestas a violencia, por sus parejas íntimas, sufrieron de maltratos psicológicos en el 100\% de los casos (Lara et al., 2019) seguidos de maltratos físicos, violencia sexual, violencia económica patrimonial, incluida violencia religiosa; siendo que en el Cercado de Lima, en el 2019, se registraron 561 denuncias por violencia familiar de las cuales, el 36\% correspondieron a mujeres entre los 18 a 29 años de edad, el 34\% fueron mujeres entre los 30 a 59 años de edad, seguido por $11.05 \%$ de casos de mujeres mayores de 60 años, reportándose un porcentaje menor de casos de menores entre 1 a 17 años que equivalieron al 3.38\%, siendo la violencia psicológica la de mayor prevalencia con un $72.37 \%$ seguida de la violencia física con un $7.48 \%$, existiendo denuncias por ambas agresiones que llegaron a un 16.58\% (DIVPCVF, diciembre 2019).

De modo similar, se encontró el grupo de las mujeres embarazadas que en un $41,4 \%$ tienen signos depresivos (Da Silva et al., 2020), siendo que la edad, los ingresos familiares, el comienzo a la etapa sexual, enfermedad durante el embarazo, el deseo de interrumpirlo y el número de parejas fueron factores asociados a la violencia psicológica (Ranielle y Franciéle, 2020). La prevalencia de violencia psicológica en los casos de mujeres y de aquellas que se encuentran gestando, se corroboró con las estadísticas presentadas por el MINP, en su boletín de noviembre 2020, que señala que, de 58595 mujeres sometidas a violencia familiar, entre los 18 y 59 años de edad, el 49.8\% era por violencia psicológica. Al respecto, la mayor incidencia de hechos violentos a nivel social e interpersonal, así como el incremento en la aparición de signos depresivos son algunos de los problemas psicosociales que se han constituido en importantes problemas de salud pública en los inicios del presente siglo (Arellanez y Cortés, 2018).

Se evidenció que los niños también se ven afectados en su salud mental, debido a las agresiones psicológicas que les ocasionan sus padres (Cleto et al., 2019), acarreándoles bajo rendimiento escolar e introspección, haciéndolos vulnerables al consumo de drogas (Brock et al., 2017). Por su parte, los adolescentes que sufrieron de falta de apoyo o conflictos familiares, sus procesos psicosociales se vieron dañados, convirtiéndose en un factor de riesgo para síntomas depresivos u otras conductas autodestructivas. En ese sentido, el MINSA informó que los trastornos más habituales en infantes y jóvenes son por problemas emocionales, como depresión o ansiedad ligados a violencia doméstica, mientras que en el Instituto Nacional de Salud Mental 
prevaleció los trastornos depresivos asociados a ideas suicidas, revelándose que hay un $20 \%$ o $25 \%$ de jóvenes que tienen deseos de morir de los cuales un 10\% o 15\% quieren hacerlo realidad (Sausa, 2018).

Algo semejante ocurre con los adultos mayores, como integrantes del grupo familiar, no escapan de sufrir de algún tipo de violencia, y sobre todo de la psicológica, siendo los más afectados los adultos entre los 70 y 79 años de edad, que en un porcentaje del 56.7\% presentaron signos de depresión (García et al., 2016), de igual forma, el Ministerio de la Mujer y Poblaciones Vulnerables (MIMP, 2020) informó que de 6791 (100\%) casos de ancianos víctimas de agresión familiar, 4439 (65.37\%) de ellos, fueron atendidos por violencia psicológica. En este orden de ideas, podemos deducir claramente que la violencia intrafamiliar, en su modalidad de violencia psicológica, se encuentra asociada o es causante de trastornos depresivos en las mujeres y en los miembros del grupo familiar, haciéndose necesario políticas de salud pública que permitan desarrollar mejores acciones preventivas y brindar una adecuada atención a las víctimas de este tipo de violencia, así como sensibilizar a la población para que denuncien oportunamente a las autoridades cuando tomen conocimiento de algún caso de violencia familiar y no se vuelvan cómplices silenciosos de este flagelo.

\section{CONCLUSIONES}

De los artículos revisados se evidenció claramente una correlación entre los trastornos psicológicos a casusa de la violencia infringida a las mujeres por sus parejas íntimas, quienes presentaron cuadros severos de depresión. En la etapa de gestación se presentó un elevado porcentaje de trastornos depresivos relacionados con la violencia ejercida por la pareja, siendo la violencia psicológica la más prevalente entre las mujeres jóvenes embarazadas de bajos recursos económicos, poca educación y que en algunos casos querían interrumpir su embarazo.

La merma en el bienestar mental de las mujeres se incrementa cuando se sienten culpables de exponer a sus hijos a la violencia familiar. Las diferentes formas de violencias ejercidas dentro del hogar, suscitan afectación psicológica a los componentes del núcleo familiar en especial a los infantes y jóvenes, siendo notaria la injerencia de la violencia familiar en la aparición de cuadros depresivos de los adolescentes.

La planificación de vida y desarrollo social (condición civil, instrucción, niveles de vida y rentas familiares) se tornan desfavorables para las adolescentes embarazadas que han sido violentadas. En tanto que, los ancianos o adultos mayores, indistintamente de su sexo, son más vulnerables a la agresión psicológica, apatía y desatención por parte de los otros miembros del grupo familiar.

\section{REFERENCIAS BIBLIOGRAFICAS}

Arellanez, J., y Cortés, E. (2018). Resultados de una intervención sobre prevención de la violencia familiar, depresión, ideación suicida y consumo de drogas en estudiantes de bachillerato. Eduscientia, 119. https://www.mendeley.com/reference-manager/reader/dba178c4-afb8-3be0-ae68-02873799918a/7bd31 694-4d39-bf69-27bb-8971a8d6eeca

Baena, G., Carmona, J., y Rengifo, C. (2020). Propuesta de intervención sobre la violencia intrafamiliar: abordaje de acuerdo con la función y sentido del fenómeno violento presente en la dinámica familiar. Estudos de Psicologia (Campinas), 37. https://doi.org/10.1590/1982-0275202037e180104

Bonino, L., y Corsi, J. (2003). Violencia y género: la construcción de la masculinidad como factor de riesgo. In Violencias Sociales (Ed.), Ariel. Ariel. https://dialnet.unirioja.es/servlet/articulo?codigo=981728

Brock, J., Pereira, N., Matheus, F., Damasceno, J., Santos, R., y Lorenzini, A. (2017). Violência conjugal: repercussões para mulheres e filhas(os). Escola Anna Nery, 21(4). https://doi.org/10.1590/2177-9465-ean-2016-0346

Cleto, M., Covolan, N., y Signorelli, M. (2019). Mulheres-mães em situação de violênciadoméstica e familiar no contexto do acolhimentoinstitucional de seus(as) filhos(as): o paradoxo daproteção integral. Saude e Sociedade, 28(3), 157-170. https://doi.org/10.1590/s0104-12902019170922 
Cudris, L., Guzmán, C., González, A., Silvera, L., y Bolaño, L. (2019). Malestar psicológico en víctimas de violencia sexual, intrafamiliar y del conflicto armado. Tempus Psicológico, 3(1), 81-102. https://doi.org/10.30554/temp uspsi.3.1.2878.2020

Da Silva, L., Amaral do Carmo, T., Custodio, B., y Da Cunha, J. (2020). Sintomas depressivos em gestantes e violência por parceiro íntimo: um estudo transversal. Enfermería Global, 19(4), 1-45. https://doi.org/10.6018/eglobal. 408841

División de Protección contra la Violencia Familiar. DIVPCVF. (diciembre, 2019). Denuncias registradas en la Comisaría PNP de la Familia - Lima, correspondiente al año 2019.

García, R., Guisado, K., y Torres, A. (2016). Maltrato intrafamiliar hacia el adulto mayor en el del Policlínico Reynold Garcia de Versalles. http://scielo.sld.cu/scielo.php?script=sci_arttextypid=S1684-18242016000600004

Lara, E., Aranda, C., Zapata, R., Bretones, C., y Alarcón, R. (2019). Depresión y ansiedad en mujeres víctimas de violencia en la relación de pareja. Revista Argentina de Ciencias Del Comportamiento, ISSN 1852.4206. https://www.mendeley.com/reference-manager/reader/13a42c29-7d3d-3156-a09b-b6f3319f9ad 3/3afcac03-71c0-6c5f-37f1-f9f0c89ea776

Ley N. 30364. (2015). Ley para prevenir, sancionar y erradicar la violencia contra las mujeres y los integrantes del grupo familiar.

MIMP, y Ministerio de la Mujer y Poblaciones Vulnerables. (2020). Programa Nacional para la Prevención y Erradicación de la Violencia contra las Mujeres e Integrantes del Grupo Familiar - AURORA. https://www.mim p.gob.pe/files/programas_nacionales/pncvfs/estadistica/boletin_noviembre_2020/BV_Noviembre_2020.pdf

Olivera, A., Rivera, E., Gutiérrez, M., y Méndez, J. (2019). Funcionalidad familiar en la depresión de adolescentes de la Institución Educativa Particular "Gran Amauta de Motupe" Lima, 2018. Revista Estomatológica Herediana, 29(3), 189-195. https://doi.org/10.20453/reh.v29i3.3602

OMS, y Organización Mundial de la Salud. (2020, January 30). Depresión. Centro de Prensa.

Orchiucci, P., De La Plata, L., Salcedo, D., Yoshikawa, E., y Monteiro, C. (2020). Adolescence, pregnancy and domestic violence: social conditions and life projects. Revista Brasileira de Enfermagem, 73 1, e20190111. https://doi.or $\mathrm{g} / 10.1590 / 0034-7167-2019-0111$

Ranielle, S., y Franciéle, L. (2020). Intimate partner violence during pregnancy: prevalence and associated factors. Revista de Saude Publica, 54, 97. https://doi.org/10.11606/s1518-8787.2020054002103

Saldaña, H., y Gorjón, G. (2020). Causas y consecuencias de la violencia familiar: caso Nuevo León. Justicia, 25, $243-$ 268. https://doi.org/10.17081/just.25.38.4002

Sausa, M. (2018, February 3). Menores ocupan el 70\% de atenciones en salud mental [INFOGRAFÍA]. Perú 21. htt ps://peru21.pe/peru/cifras-salud-mental-peru-menores-ocupan-70-atenciones-infografia-394376-noticia/

\section{INFORMACIÓN ADICIONAL}

Aspectos éticos: Declaran los autores que han respetado lo establecido por las normas éticas que regulan el ejercicio profesional

Conflicto de intereses: Declaran los autores que no han incurrido en conflicto de intereses al elaborar el presente artículo. 